\title{
Comparison of belief AHP models using a simulation study
}

\author{
Amel Ennaceur ${ }^{1}$ Zied Elouedi $^{1}$ Eric Lefevre $^{2}$ \\ ${ }^{1}$ LARODEC, University of Tunis, Institut Supérieur de Gestion, Tunisia \\ ${ }^{2}$ Univ. Lille Nord of France, UArtois EA 3926 LGI2A, France
}

\begin{abstract}
A multi-criteria decision making (MCDM) method, as its own name suggests, is for use in situations when more than one criterion must be considered. In this work, we will focus on the Analytic Hierarchy Process (AHP), one of the well-known and most widely used MCDM approaches. Despite the extensive application of this method in various fields of decision making, AHP has been combined within uncertain theories, in particular the belief function theory, to handle uncertainty in the pair-wise comparison process. Therefore, this paper intends to describe some comparisons on the standard AHP and other belief AHP models to judge their accuracy. We use a simulation approach to compare the results of the different models based on different matrices dimensions. We observe a high level of agreement between the different techniques and we can conclude that the number of ranking contradictions increases with the dimension of the matrix.
\end{abstract}

Keywords: AHP, uncertain AHP, qualitative AHP, random data, simulation

\section{Introduction}

Multi-Criteria Decision Making (MCDM) is an area of Operational Research that supports the process of decision making. In this field, the goal is to rank different alternatives considering multiple, often conflicting, criteria. Thus, this discipline is gaining importance as potential tools for analyzing complex real problems due to their inherent ability to judge different alternatives on various criteria for possible selection of the best/suitable alternative(s). These alternatives may be further explored in-depth for their final implementation [1].

Consequently, several methods have been proposed for solving MCDM problems [2] [3] [4]. A major criticism of MCDM is that several techniques may yield different results when applied to the same problem. In fact, many methods may appear to be suitable for a particular decision problem. Hence, the user faces the task of selecting the most appropriate method from among several feasible approaches. Therefore, this paper tries to give enough information for the decision maker to decide, which is the most appropriate one to use according to his liking and the main characteristics of the problem.
Besides, no model is considered to be better than another, therefore, we cannot say than one method gives more reliable result than another. In fact, the same problem yields different results according to several methods, this is explained by the different assumptions that distinguish one method from another. In addition, not all MCDM problems are similar. Some have very detailed and quantitative information with little subjectivity. Others have a lot of subjectivity in their nature. Some others have a blend of quantitative and qualitative data, a mixture of objective and subjective information. According to the expert experience, we can then choose the appropriate model.

One of the well-known and most widely used MCDM approaches is the Analytic Hierarchy Process (AHP) [3] [4]. Despite the extensive application of this method in various fields of decision making, this method has been criticized since it gives good results in a context in which everything is known with certainty. However, the reality is connected to uncertainty and imprecision by nature. Hence, one of the main problems of the standard AHP is that it does not take into account uncertainty in the judgments since the matrices of judgments are deterministic. In real applications, the decision maker is always subject to uncertainty while expressing their judgments and do not like to be forced to give deterministic answers. Moreover, it is also recognized that human assessment on qualitative attributes is always subjective and thus imprecise. This limitation greatly reduces the confidence of the users on the final results of the AHP technique.

In order to model this kind of uncertainty, several AHP methods are combined within uncertain theories [5] [6]. In this paper, we will especially focus on AHP method under the belief function framework. Variants of methods come into implementation such as: [7] [6]. In this work, we will present uncertain AHP which represents decision maker assessments using a new elicitation procedure [8]. Also, qualitative AHP models expert preference using preference relations [9]. The main difference between the three models is the pair-wise comparison technique. In fact, they have the same skeleton; however they use different preference elicitation techniques.

These belief decision-making methods can all be used in belief decision-making problems. However, they may derive different answers for the same prob- 
lem. Since the best alternative should be the same no matter which method is used, an examination of the accuracy and consistency of these methods is highly desirable. So, our main goal is then to compare and contrast these three approaches, AHP, qualitative $\mathrm{AHP}$ and uncertain $\mathrm{AHP}$, to decision aiding from both a theoretical and practical standpoint. The strengths and weaknesses of each will be commented on. This will enable a decision maker or analyst to perceive more clearly the choice to make between the three approaches and the implications of such a choice.

In what follows, we first present some definitions needed for belief function context. Next, we describe the uncertain and qualitative AHP methods in section 3 . Then, section 4 details the evaluation algorithm. Finally, section 5 concludes the paper.

\section{Belief Function Theory}

\subsection{Basic Concepts}

The Transferable Belief Model (TBM) is a model to represent quantified belief functions [10]. Let $\Theta$ be the frame of discernment representing a finite set of elementary hypotheses related to a problem domain. We denote by $2^{\Theta}$ the set of all the subsets of $\Theta[11]$.

The impact of a piece of evidence on the different subsets of the frame of discernment $\Theta$ is represented by the so-called basic belief assignment (bba), denoted by $m[11]$ :

$$
\sum_{A \subseteq \Theta} m(A)=1
$$

The value $m(A)$, named a basic belief mass (bbm), represents the portion of belief committed exactly to the event $A$. The events having positive bbm's are called focal elements. Let $\mathcal{F}(m) \subseteq 2^{\Theta}$ be the set of focal elements of the bba $m$.

Associated with $m$ is the belief function is defined for $A \subseteq \Theta$ and $A \neq \emptyset$ as:

$$
\operatorname{bel}(A)=\sum_{\emptyset \neq B \subseteq A} m(B) \text { and } \operatorname{bel}(\emptyset)=0
$$

The degree of belief $\operatorname{bel}(A)$ given to a subset $A$ of the frame $\Theta$ is defined as the sum of all the basic belief masses given to subsets that support $A$ without supporting its negation.

\subsection{Uncertainty Measures}

In the case of the belief function framework, the bba is defined on an extension of the powerset: $2^{\Theta}$ and not only on $\Theta$. In the powerset, each element is not equivalent in terms of precision. Indeed, $\theta_{i} \subset \Theta$ $(i \in\{1,2\})$ is more precise than $\theta_{1} \cup \theta_{2} \subseteq \Theta$.

In order to try to quantify this imprecision, different uncertainty measures have been defined, such as [12]:

$$
H(m)=\sum_{A \in \mathcal{F}(m)} m(A) \log _{2}\left(\frac{|A|}{m(A)}\right) .
$$

\subsection{Decision Making}

The TBM considers that holding beliefs and making decision are distinct processes. Hence, it proposes a two level model [13]: The credal level where beliefs are entertained and represented by belief functions, and the pignistic level where beliefs are used to make decisions and represented by probability functions called the pignistic probabilities, denoted $\operatorname{BetP}[13]$ :

$$
\operatorname{BetP}(A)=\sum_{B \subseteq \Theta} \frac{|A \cap B|}{|B|} \frac{m(B)}{(1-m(\emptyset))}, \forall A \in \Theta
$$

\section{MCDM Method under the Belief Function Framework}

Of the many MCDM methods available, we have chosen the following three models for comparison in our research, when applied to solve the same problem. Uncertain AHP and qualitative AHP [8] [14] have the same skeleton as standard AHP method. Therefore, building a qualitative AHP or uncertain AHP falls to the definition of its fundamental steps, namely, hierarchical model, pair-wise comparisons, local priorities and global priorities. These concepts must take into account the uncertainty encountered in the obtained data. Thus, the main difference between these three approaches is how to model and to quantify expert assessments. In other words, these approaches handle uncertainty in the pair-wise comparison process. AHP transform expert preferences into quantitative using $1-9$ scale. Uncertain AHP represents decision maker assessments using a new set of choice based on yes or no. While, qualitative AHP models expert judgments using preference relations.

\subsection{AHP method}

The Analytic Hierarchy Process (AHP) is a multicriteria decision-making approach and was introduced by Saaty [3] [4]. AHP organizes the basic rationality by breaking down a problem into its smaller constituent parts. By decomposing the problem, the decision-maker can focus on a limited number of items at the same time. The AHP is carried out in two phases: the design of the hierarchy and the evaluation of the components in the hierarchy [4]. It involves building a hierarchy of decision elements and then making comparisons between each possible pair (as a matrix).

In a pair-wise comparison, the decision maker examines two alternatives by considering one criterion and indicates a preference. These comparisons are 
made using a preference scale, which assigns numerical values to different levels of preference. The standard preference scale used for AHP is $1-9$ scale which lies between "equal importance" to "extreme importance" where sometimes different evaluation scales can be used such as 1 to 5 . In the pair-wise comparison matrix, the value 9 indicates that one factor is extremely more important than the other, and the value $1 / 9$ indicates that one factor is extremely less important than the other, and the value 1 indicates equal importance. Therefore, if the importance of one factor with respect to a second is given, then the importance of the second factor with respect to the first is the reciprocal. Ratio scale and the use of verbal comparisons are used for weighting of quantifiable and non-quantifiable elements.

\subsection{Uncertain AHP method}

Under this approach [8], a new elicitation procedure, which incorporates belief distributions to include uncertainty in the judgments, is introduced. Thus to model his assessments, the decision maker has to express his opinions qualitatively. He indicated whether a criterion (or alternative) was more or less important to its partner by "yes" or "no". Moreover, the expert may define uncertain or even unknown assessments. Indeed, each subset of criteria is described by a basic belief assignment defined on the possible responses.

The computational procedure can be summarized as follows:

1. Model the problem as a hierarchy where $\Theta=$ $\left\{a_{1}, \ldots, a_{m}\right\}$ is the set of alternatives and $\Omega=$ $\left\{c_{1}, \ldots, c_{n}\right\}$ is the group of criteria.

2. Establish priorities among the elements of the hierarchy by making a series of judgments based on pair-wise comparisons of the elements using the "yes" or "no" model. Each assessment is described by a basic belief assignment to quantify the degree of beliefs.

3 . For each pair-wise comparison matrix, we denote, for example, $m_{j}^{\Omega c_{i}}$ the importance of $c_{i}$ with respect to the criterion $c_{j}$ (for simplicity we denote the criterion by $j$ instead of $c_{j}$ ).

4. For each row of the matrix, we apply the conjunctive rule. That means, for each criterion $(i=\{1, \ldots, n\})$, we will get the following bba:

$$
m^{\Omega_{c_{i}}}=@ m_{j}^{\Omega_{c_{i}}} \text {, where } j=\{1, \ldots, n\}
$$

5. Rather than the standard consistency index, we propose here to check the conflict in expert judgments. Therefore, if the opinions are far from each other, we consider that they are in conflict and the expert has to reformulate his evaluations.

6. The obtained bba $m^{\Omega_{c_{i}}}$ is transformed into pignistic probabilities, denoted by $\operatorname{Bet} P^{\Omega_{c_{i}}}$.

7. At the alternative level, the same process is repeated regarding each criterion (we use the same pair-wise comparison technique). The purpose is then to combine these obtained bba's to get a single belief function. The idea is to standardize all the frames of discernment. Obviously, we propose to use the concept of refinement operations [11] which allows us to establish relationships between different frames of discernment in order to express beliefs on anyone of them. The objective consists in obtaining one frame of discernment $\Theta$ from the set $\Theta_{a_{k}}$ by splitting some or all of its events. As mentioned above, each bba $m^{\Theta_{a_{k}}}$ represents the belief over all possible answers (yes or no). However, at this stage, we want to know which alternative is the best $\left(\Theta=\left\{a_{1}, \ldots, a_{m}\right\}\right)$. As a result, $\Theta_{a_{k}}$ is considered as a coarsening of $\Theta$, and we get the following relation:

$$
m^{\Theta_{a_{k}} \uparrow \Theta}\left(\rho_{k}(\omega)\right)=m^{\Theta_{a_{k}}}(\omega) \quad \forall \omega \subseteq \Theta_{a_{k}}
$$

where the mapping $\rho_{k}$ from $\Theta_{a_{k}}$ to $\Theta$ is a refine$\underline{\text { ment }}$, and $\rho_{k}(\{y e s\})=\left\{a_{k}\right\}$ and $\rho_{k}(\{n o\})=$ $\overline{\left\{a_{k}\right\}}$.

Once we have standardized our frame of discernment $\Theta$, we can now combine the resulting bba using the conjunctive rule to get $m_{c_{k}}($.$) .$

8. To update the alternatives priorities with respect to the criterion weight, this approach proposes to regard each pignistic probability distribution of a specific of criterion as a measure of reliability. This factor is used to measure most heavily the bba evaluated according to the most important criteria and conversely for the less important ones. If we have $c_{k}$ a defined criterion, then we get $\beta_{k}$ its corresponding measure of reliability:

$$
\operatorname{Bet} P^{\Omega_{c_{k}}}(\{y e s\})=\beta_{k} \text { and } \operatorname{Bet} P^{\Omega_{c_{k}}}(\{n o\})=1-\beta_{k}
$$

As a result, the corresponding bba will be directly discounted:

$$
\begin{aligned}
& m_{c_{k}}^{\alpha_{k}}\left(a_{j}\right)=\beta_{k} \cdot m_{c_{k}}\left(a_{j}\right), \forall a_{j} \subset \Theta \\
& m_{c_{k}}^{\alpha_{k}}(\Theta)=\left(1-\beta_{k}\right)+\beta_{k} \cdot m_{c_{k}}(\Theta)
\end{aligned}
$$

where $m_{c_{k}}\left(a_{j}\right)$ the relative bba for the subset $a_{j}$ (obtained in the previous step), $\beta_{k}$ its corresponding measure of reliability, and we denote $\alpha_{k}=1-\beta_{k}$.

9. Combine the overall bba's to get a single representation by using the conjunctive rule.

10. Come to a final decision based on the pignistic transformation to choose the best alternative. The decision maker will choose the alternative which has the highest value.

\subsection{Qualitative AHP Method}

Ennaceur et al. [9] propose a revised version of the AHP model. They demonstrate that standard AHP 
is thought to be a robust way to solve determined decision making problem. However, it neglects the uncertainty caused by subjective preference of decision maker in criteria and alternative scoring. Its pair-wise comparison value seems not strong enough to cover most decision makers' options. Accordingly, uncertain AHP was used to improve this situation.

Therefore, in the proposed methodology, the expert is allowed to use preference relations only. Thus, to express his assessments, the decision maker has to express his opinions qualitatively, based on knowledge and experience that he provides in response to a given question rather than direct quantitative information. He only selects the related linguistic variable using preference modeling. The preference relations may be: a preference relation $(\succ)$, an indifference relation $(\sim)$, a weak preference relation $(\succeq)$, or an unknown relation $(-)$.

To present the uncertain AHP method, we introduce its different construction steps, described as follows:

1. Model the problem as a hierarchy containing the decision goal, the sets of alternatives $\Theta=$ $\left\{a_{1}, \ldots, a_{m}\right\}$ for reaching it, and the sets of criteria $\Omega=\left\{c_{1}, \ldots, c_{n}\right\}$ for evaluating the sets of alternatives.

2. Establish priorities among the elements of the hierarchy by making a series of judgments based on pair-wise comparisons of the elements using only preference relations.

3 . For each pair-wise comparison matrix, transform preference relations into numerical values using the belief function theory. Therefore, Ennaceur et al. model $[14,15]$ is applied to convert these preferences into constraints of an optimization problem whose resolution, according to some uncertainty measures (UM). This model allows the generation of the least informative or the most uncertain belief functions. It can then be determined by the resolution of an optimization problem. For instance, if we use the preference relations matrix relative to the criterion level we get:

$$
\begin{gathered}
\operatorname{Max} H(m)=m\left(\left\{c_{1}\right\}\right) * \log _{2}\left(\left|c_{1}\right| / m\left(\left\{c_{1}\right\}\right)\right)+ \\
m\left(\left\{c_{2}\right\}\right) \log _{2}\left(\left|c_{2}\right| / m\left(\left\{c_{2}\right\}\right)\right)+\ldots+ \\
m\left(\left\{c_{n}\right\}\right) * \log _{2}\left(\left|c_{n}\right| / m\left(\left\{c_{n}\right\}\right)\right)+ \\
m(\Omega) * \log _{2}(|\Omega| / m(\Omega)) ; \\
\text { s.t. } \\
\operatorname{bel}\left(\left\{c_{i}\right\}\right)-\operatorname{bel}\left(\left\{c_{j}\right\}\right) \geq \gamma \quad \forall\left(c_{i}, c_{j}\right), c_{i} \succ c_{j} \\
\operatorname{bel}\left(\left\{c_{i}\right\}\right)-\operatorname{bel}\left(\left\{c_{j}\right\}\right) \leq \gamma \quad \forall\left(c_{i}, c_{j}\right), c_{i} \succeq c_{j} \\
\operatorname{bel}\left(\left\{c_{i}\right\}\right)-\operatorname{bel}\left(\left\{c_{j}\right\}\right) \geq \varepsilon \quad \forall\left(c_{i}, c_{j}\right), c_{i} \succeq c_{j} \\
\operatorname{bel}\left(\left\{c_{i}\right\}\right)-\operatorname{bel}\left(\left\{c_{j}\right\}\right) \geq-\varepsilon \quad \forall\left(c_{i}, c_{j}\right), c_{i} \sim c_{j} \\
\operatorname{bel}\left(\left\{c_{i}\right\}\right)-\operatorname{bel}\left(\left\{c_{j}\right\}\right) \leq \varepsilon \quad \forall\left(c_{i}, c_{j}\right), c_{i} \sim c_{j} \\
\ldots \\
\sum_{c_{i} \in \mathcal{F}(m)} m\left(c_{i}\right)=1, m(A) \geq 0, \forall A \subseteq \Omega ; m(\emptyset)=0 .
\end{gathered}
$$

Where $H$ is the uncertainty measure since it has a unique maximum. Besides, it takes into account the non-specificity and quantifies the conflict presented in the body of evidence (measure of total uncertainty). Besides, the preference relations are transformed into constraints as follows: the first constraint of the model is derived from the preference relation. The second and third constraints model the weak preference relation. The fourth and fifth constraints correspond to the indifference relation. Each preference relation must be translated into one of the presented constraint.

$\varepsilon$ and $\gamma$ are a constant specified by the expert before beginning the optimization process.

4. Assume that the priorities of criteria and alternatives are described by a basic belief assignment defined on the possible responses. Thus, the criterion bba is denoted by $m^{\Omega}$ and the alternative bba by $m^{\Theta}$.

5. At the criterion level, the obtained bba is transformed into measure of reliability. If we have $c_{i}$ as a criterion, then we get $\beta_{i}$ its corresponding measure of reliability:

$$
\beta_{i}=\frac{m^{\Omega}\left(c_{i}\right)}{\max _{k} m^{\Omega}\left(c_{k}\right)}
$$

6. Synthesize the overall judgment, that is updating the sets of alternatives priorities with the importance of their corresponding criteria. We use the same aggregation process as presented in uncertain AHP. So, we apply Equations 8 and 9 to update the alternative bbas.

7. Combine the overall bba's to get a single representation by using the conjunctive rule.

8. Come to a final decision based on the the pignistic transformation to choose the best alternatives. Besides, we can compute the pignistic probabilities to choose the best alternatives (Equation 4).

\section{Computational experiments}

In this section, the performances of the three presented methods, AHP, uncertain AHP and qualitative AHP are compared. Firstly, the evaluation algorithm used in the comparisons are described. Secondly, the methods are tested on randomly generated matrices. Then, we discuss the obtained result. And lastly, proposed methods are compared based on an example that is commonly used in the literature.

\subsection{Evaluation algorithm}

Despite the possible differences between the obtained results of three MCDM methods, we cannot conclude the superiority of one over another. Unless we have a solid basis, we compare the ranking results against the closeness of the rankings of each method to the actual rankings. To do so, we need to compare each set of rankings provided by AHP 
and the proposed methods with a ranking that has been already produced by an alternative, yet reliable ranking method. This alternative ranking will be considered as a basis, or actual ranking of the alternatives and will be used to measure the closeness of the rankings provided by AHP, uncertain AHP and qualitative AHP to reality.

A method is accurate in MCDM problems should also be accurate in single dimensional problems. Therefore, we use the Weighted Sum Model (WSM) method, since in single-dimensional environment, it yields the most reasonable results. Hence, Triantaphyllou et al. [16, 17] compare the obtained results using WSM by those obtained by other MCDM methods. This evaluation criterion has been applied in order to evaluate crisp and fuzzy MCDM methods $[16,17]$.

Besides, WSM is the simplest and still the widest used MCDM method. In this method, each criterion is given a weight and the sum of all weights must be 1. Each alternative is assessed with regard to every criterion. The overall or composite performance score of an alternative is given by the equation:

$$
P_{i}=\sum_{j} v_{i j} * \omega_{j}
$$

where $P_{i}$ is the priority of each alternative, $\omega_{j}$ is the weight of each criterion and $v_{i j}$ is the score of each alternative regarding each criterion.

In order to overcome these issues, in the next section, we test a simulation algorithm that compares the ranking results of the three methods under different scenarios.

\subsection{Simulation algorithm}

To generate reliable data for a numerical analysis in AHP, simulation has been extensively used in prior research $[17,16]$.

The experiment is based on the following steps:

1. We generate a random matrix for the decision performance as well as a random matrix to represent the weight of each decision criteria. Based on these two matrices, the overall scores and ranks of the decision alternatives are calculated. These steps are usual steps in the WSM method.

2. From the performance matrix, we generated pair-wise comparison matrices of different alternatives that are compared to each criterion.

3. We apply the suggested method to compute the overall priorities and to rank alternatives.

4. We compare the obtained result with the ranking of the WSM method.

\subsubsection{Example 1}

Let's demonstrate the evaluation procedure using the same example used in Triantaphyllou et al. [16]. We consider 3 alternatives $a_{1}, a_{2}$ and $a_{3}$ and three criteria $c_{1}, c_{2}$ and $c_{3}$. The decision making problem is described using the matrix presented in Table 1.

Table 1: Decision matrix
\begin{tabular}{cccc}
\multicolumn{4}{c}{ Criteria } \\
\hline & $c_{1}$ & $c_{2}$ & $c_{3}$ \\
\hline & $\frac{8}{13}$ & $\frac{2}{13}$ & $\frac{3}{13}$ \\
$a_{1}$ & 1 & 9 & 9 \\
$a_{2}$ & 5 & 2 & 2 \\
$a_{3}$ & 1 & 5 & 9 \\
\hline
\end{tabular}

This example has been solved using the WSM and AHP in [16]. While applying the WSM, it can be shown that the alternative $a_{1}$ is the best one and AHP it turns out that the alternative $a_{2}$ is the best one. Obviously, this is in contradiction with the conclusion derived using the WSM.

Now, let us model this example using uncertain AHP. If the decision maker knew the actual data shown in the original crisp decision matrix, then the matrix of the actual pair-wise comparisons when the three alternatives would be compared regarding each criterion will be illustrated in Table 2 .

Next, and after modeling pair-wise comparison matrices using Saaty scale, we transform them into belief pair-wise comparison. Indeed, the resulting bba's has 2 focal elements:

- The first is the actual preference's value regarding the alternative $a$ with bbm, $m(a)=p * 0.1$ (In our case $p$ is obtained from the classical Saaty's scale comparison).

- The second is $\Theta$ such as $m(\Theta)=1-m(a)$

We obtain the results as shown in Figure 1.

We use the uncertain AHP method to aggregate the obtained priorities. We obtain the following order: $a_{1}>a_{2}>a_{3}$. Obviously, this is in contradiction with the results derived when the AHP method was applied at the beginning of this illustrative example. However, we have identified the same best alternative as WSM.

Now, let us model this example using qualitative AHP. If the decision maker knew the actual data shown in the original crisp decision matrix, then the qualitative matrix of the actual pair-wise comparisons would be obtained as follows:

- "Equal importance" is equivalent to indifference relation.

Table 2: The pair-wise comparison matrices

\begin{tabular}{|c|c|c|c|}
\hline \multicolumn{4}{|c|}{$c_{1}$} \\
\hline & $a_{1}$ & $a_{2}$ & $a_{3}$ \\
\hline$a_{1}$ & - & $\frac{1}{5}$ & 1 \\
$a_{2}$ & - & - & 5 \\
$a_{3}$ & - & - & - \\
\hline
\end{tabular}

\begin{tabular}{|c|c|c|c|}
\hline \multicolumn{4}{|c|}{$c_{2}$} \\
\hline & $a_{1}$ & $a_{2}$ & $a_{3}$ \\
\hline$a_{1}$ & - & $\frac{9}{2}$ & $\frac{9}{5}$ \\
$a_{2}$ & - & - & $\frac{2}{5}$ \\
$a_{3}$ & - & - & - \\
\hline
\end{tabular}

\begin{tabular}{|c|c|c|c|}
\hline \multicolumn{5}{|c|}{$c_{3}$} \\
\hline & $a_{1}$ & $a_{2}$ & $a_{3}$ \\
\hline$a_{1}$ & - & $\frac{9}{2}$ & 1 \\
$a_{2}$ & - & - & $\frac{2}{9}$ \\
$a_{3}$ & - & - & - \\
\hline
\end{tabular}




\begin{tabular}{|c|c|c|c|}
\hline$c_{1}$ & $\left\{a_{1}\right\}$ & $\left\{a_{2}\right\}$ & $\left\{a_{3}\right\}$ \\
\hline$\left\{a_{1}\right\}$ & $m_{\left\{a_{1}\right\}}^{\Omega_{\left\{a_{1}\right\}}}\left(\Omega_{\left\{a_{1}\right\}}\right)=1$ & $\begin{array}{l}m_{\left\{a_{2}\right\}}^{\Omega_{\left\{a_{1}\right\}}}(\{\text { yes }\})=0.02 \\
\Omega_{\left\{a_{1}\right\}}\left(\Omega_{\left\{a_{1}\right\}}\right)=0.98 \\
m_{\left\{a_{2}\right\}}\end{array}$ & $\begin{array}{l}m_{\left\{a_{3}\right\}}^{\Omega_{\left\{a_{1}\right\}}}(\{\text { yes }\})=1 \\
\Omega_{\left\{a_{1\}}\right\}}\left(\Omega_{\left\{a_{1}\right\}}\right)=0 \\
m_{\left\{a_{3}\right\}}\end{array}$ \\
\hline$\left\{a_{2}\right\}$ & $\begin{array}{c}m_{\left\{a_{1}\right\}}^{\Omega_{\left\{a_{2}\right\}}}(\{n o\})=0.02 \\
m_{\left\{a_{2}\right\}}\left(\Omega_{\left\{a_{2}\right\}}\right)=0.98\end{array}$ & $m_{\left\{a_{2}\right\}}^{\Omega_{\left\{a_{2}\right\}}}\left(\Omega_{\left\{a_{2}\right\}}\right)=1$ & $\begin{array}{l}\left.m_{\left\{\left\{a_{2}\right\}\right.}^{\Omega(y e s\}}\right)=0.5 \\
m_{\left\{a_{3}\right\}} \\
m_{\left\{a_{2}\right\}}\left(\Omega_{\left\{a_{2}\right\}}\right)=0.5\end{array}$ \\
\hline$\left\{a_{3}\right\}$ & $\begin{array}{l}m_{\left\{a_{1}\right\}}^{\Omega_{\left\{a_{3}\right\}}}(\{n o\})=1 \\
m_{\left\{a_{3}\right\}}\left(\Omega_{\left\{a_{3}\right\}}\right)=0 \\
m_{\left\{a_{1}\right\}}\end{array}$ & $\begin{array}{c}m_{\left\{a_{3\}}\right\}} \underbrace{}_{\{n o\})}=0.5 \\
m_{\left\{a_{3\}}\right\}}\left(\Omega_{\left\{a_{3}\right\}}\right)=0.5\end{array}$ & $m_{\left\{a_{3}\right\}}^{\Omega_{\left\{a_{3}\right\}}}\left(\Omega_{\left\{a_{3}\right\}}\right)=1$ \\
\hline
\end{tabular}

scale). In these test problems, the number of alternatives was equal to the following values: $3,4,5,7,9$ and 11. Similarly, the number of criteria was equal to $3,4,5,6,7,10,13$ and 15 . Thus, a total of $(8 \times 6)$ different cases were examined with 100 replications (in order to derive statistically significant results) per each case. Each random problem was solved using the original, uncertain and qualitative AHP

\begin{tabular}{|c|c|c|c|}
\hline$c_{2}$ & $\left\{a_{1}\right\}$ & $\left\{a_{2}\right\}$ & $\left\{a_{3}\right\}$ \\
\hline$\left\{a_{1}\right\}$ & $m_{\left\{a_{1}\right\}}^{\Omega_{\left\{a_{1}\right\}}}\left(\Omega_{\left\{a_{1}\right\}}\right)=1$ & $\begin{array}{l}m_{\left\{a_{2}\right\}}^{\Omega_{\left\{a_{1}\right\}}}(\{y e s\})=0.45 \\
\Omega_{\left\{a_{1\}}\right\}}\left(\Omega_{\left\{a_{1}\right\}}\right)=0.55 \\
m_{\left\{a_{2}\right\}}\end{array}$ & $\begin{array}{c}m_{\left\{a_{3}\right\}} \Omega_{\left\{a_{1}\right\}} \\
\Omega_{\left\{a_{1}\right\}} \\
m_{\left\{a_{3}\right\}}\left(\Omega_{\left\{a_{1}\right\}}\right)=0.82\end{array}$ \\
\hline$\left\{a_{2}\right\}$ & $\begin{array}{c}m_{\left\{a_{1}\right\}}^{\Omega_{\left\{a_{2}\right\}}}(\{n o\})=0.45 \\
\Omega_{\left\{a_{2}\right\}} \\
m_{\left\{a_{1}\right\}}\left(\Omega_{\left\{a_{2}\right\}}\right)=0.55\end{array}$ & $m_{\left\{a_{2}\right\}}^{\Omega_{\left\{a_{2}\right\}}}\left(\Omega_{\left\{a_{2}\right\}}\right)=1$ & $\begin{array}{l}m_{\left\{a_{3}\right\}}^{\Omega\left\{a_{2}\right\}} \\
\Omega_{\left\{a_{2}\right\}}(\{\text { yes }\})=0.04 \\
m_{\left\{a_{3}\right\}}\left(\Omega_{\left\{a_{2}\right\}}\right)=0.96\end{array}$ \\
\hline$\left\{a_{3}\right\}$ & $\begin{array}{l}m_{\left\{a_{1}\right\}}^{\Omega_{\left\{a_{3}\right\}}}(\{n o\})=0.18 \\
\Omega_{\left\{a_{3}\right\}} \\
m_{\left\{a_{1}\right\}}\left(\Omega_{\left\{a_{3}\right\}}\right)=0.82\end{array}$ & $\begin{array}{l}m_{\left\{a_{2\}}\right\}}^{\Omega_{\left\{a_{3}\right\}}}(\{n o\})=0.04 \\
m_{\left\{a_{3\}}\right\}}\left(\Omega_{\left\{a_{3}\right\}}\right)=0.96 \\
m_{\left\{a_{2}\right\}}\end{array}$ & $m_{\left\{a_{3}\right\}}^{\Omega_{\left\{a_{3}\right\}}}\left(\Omega_{\left\{a_{3}\right\}}\right)=1$ \\
\hline
\end{tabular}
methods. The test problems were treated as the previous illustrative example. Any ranking irregularity was recorded.

\subsection{Simulation results}

\begin{tabular}{|c|c|c|c|}
\hline$c_{3}$ & $\left\{a_{1}\right\}$ & $\left\{a_{2}\right\}$ & $\left\{a_{3}\right\}$ \\
\hline$\left\{a_{1}\right\}$ & $m_{\left\{a_{1}\right\}}^{\Omega_{\left\{a_{1}\right\}}}\left(\Omega_{\left\{a_{1}\right\}}\right)=1$ & $\begin{array}{l}m_{\left\{a_{2}\right\}}^{\Omega_{\left\{\left(a_{1}\right\}\right.}}(\{y e s\})=0.45 \\
m_{\left\{a_{1}\right\}} \\
m_{\left\{a_{2}\right\}}\left(\Omega_{\left\{a_{1}\right\}}\right)=0.55\end{array}$ & 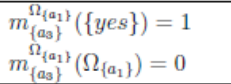 \\
\hline$\left\{a_{2}\right\}$ & $\begin{array}{l}m_{\left\{a_{1}\right\}}^{\left.\Omega_{\left\{a_{2}\right\}}\right\}}(\{n o\})=0.45 \\
m_{\left\{a_{1}\right\}} \Omega_{\left\{a_{2}\right\}}\left(\Omega_{\left\{a_{2}\right\}}\right)=0.55\end{array}$ & $m_{\left\{a_{2}\right\}}^{\Omega_{\left\{a_{2}\right\}}}\left(\Omega_{\left\{a_{2}\right\}}\right)=1$ & $\begin{array}{l}m_{\left\{a_{3}\right\}}^{\Omega\left\{\left\{a_{2}\right\}\right.}(\{y e s\}) \\
0.023 \\
\left.m_{\left\{a_{3}\right\}}\right\} \\
\left.m_{\left\{a_{3}\right\}}\right)=0.977\end{array}$ \\
\hline$\left\{a_{3}\right\}$ & $\begin{array}{l}m_{\left\{a_{3}\right\}}^{\Omega_{\left\{a_{3}\right\}}}(\{n o\})=1 \\
\Omega_{\left\{a_{3}\right\}}\left(\Omega_{\left\{a_{3}\right\}}\right)=0 \\
m_{\left\{a_{1}\right\}}\end{array}$ & $\begin{array}{l}m_{\left\{a_{2}\right\}}^{\left.\Omega_{\{} a_{3}\right\}}(\{n o\})=0.023 \\
m_{\left\{a_{3}\right\}} \\
m_{\left\{a_{2}\right\}}\left(\Omega_{\left\{a_{3}\right\}}\right)=0.977\end{array}$ & $m_{\left\{a_{3}\right\}}^{\Omega_{\left\{a_{3}\right\}}}\left(\Omega_{\left\{a_{3}\right\}}\right)=1$ \\
\hline
\end{tabular}

Figure 1: Belief pair-wise comparison matrices

Table 3: The preference relations matrices

\begin{tabular}{|c|l|l|l|}
\hline \multicolumn{4}{|c|}{$c_{1}$} \\
\hline & $a_{1}$ & $a_{2}$ & $a_{3}$ \\
\hline$a_{1}$ & - & $\prec$ & $\sim$ \\
$a_{2}$ & - & - & $\succ$ \\
$a_{3}$ & - & - & - \\
\hline
\end{tabular}

\begin{tabular}{|c|l|l|l|}
\hline \multicolumn{5}{|c|}{$c_{2}$} \\
\hline & $a_{1}$ & $a_{2}$ & $a_{3}$ \\
\hline$a_{1}$ & - & $\succeq$ & $\sim$ \\
$a_{2}$ & - & - & $\sim$ \\
$a_{3}$ & - & - & - \\
\hline
\end{tabular}

\begin{tabular}{|c|l|l|l|}
\hline \multicolumn{5}{|c|}{$c_{3}$} \\
\hline & $a_{1}$ & $a_{2}$ & $a_{3}$ \\
\hline$a_{1}$ & - & $\succeq$ & $\sim$ \\
$a_{2}$ & - & - & $\sim$ \\
$a_{3}$ & - & - & - \\
\hline
\end{tabular}

- "Somewhat more important" and "Much more important" are equivalent to weak preference relation.

- "Very much more important" and "Absolutely more important" are equivalent to strong preference relation.

As a result, the pair-wise comparison matrix of the three alternatives in terms of each criterion are illustrated in Table 3.

Next, an optimization model is used to transform preference relation into constraints and to generate quantitative information from these qualitative assessments. Finally, we obtain the following order: $a_{1} \succ a_{3} \succ a_{2}$. Obviously, this is in contradiction with the results derived when the AHP method was applied at the beginning of this illustrative example. However, we have obtained encouraging results since it can be observed that the ranking order of the alternatives as derived by the WSM and the uncertain AHP is the same.

In order to gain a deeper understanding may occur on random test problems, a computational study was undertaken. The data were random numbers from the interval $[1,9]$ (in order to be compatible with the numerical properties of the Saaty

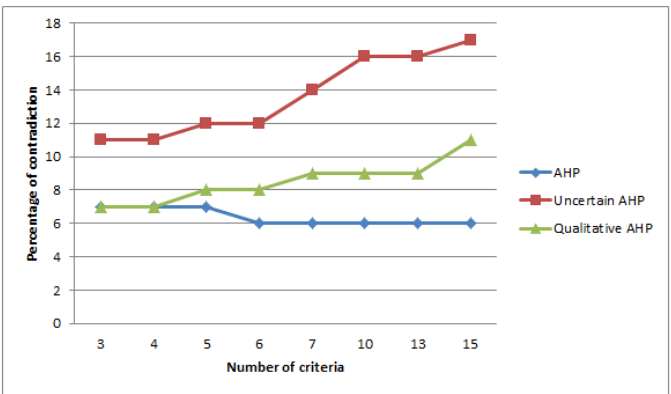

Figure 2: Percentage of contradiction (\%) based on 4 alternatives

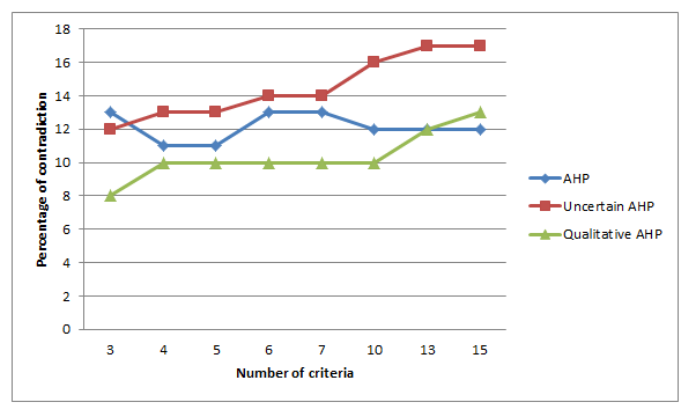

Figure 3: Percentage of contradiction (\%) based on 9 alternatives

Figure 2 and 3 refer to the use of the standard, uncertain and qualitative AHP methods. Different curves correspond to problems with different numbers of alternatives. As it can be seen from this figure, problems with few alternatives had smaller percentage of contradiction (It is the percentage of incorrect decision with reference to the best alternative obtained with WSM). Therefore, number of criteria in a problem seemed to play an insignificant role. Also, these figures show similar that the three versions of the AHP have nearly the same percentage of contradiction. However, qualitative AHP has proved the best result when it is compared according to less than 10 alternatives. 


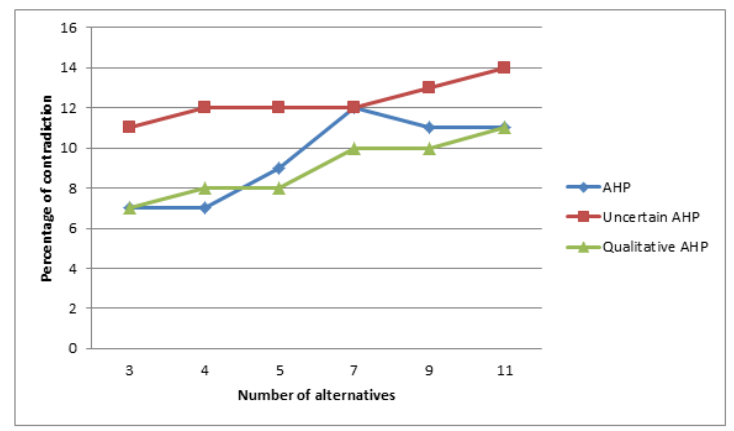

Figure 4: Percentage of contradiction (\%) based on 5 criteria

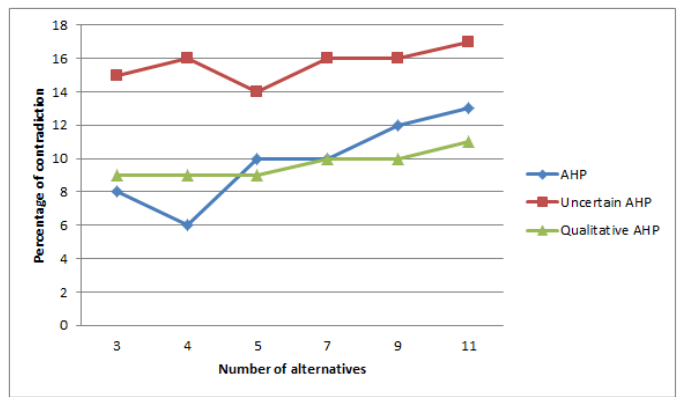

Figure 5: Percentage of contradiction (\%) based on 10 criteria

From Figures 4 and 5, we can notice that AHP and qualitative AHP methods outperform uncertain AHP. Nevertheless, we can conclude that when the number of alternatives increases (greater than 9) the percentage of contradiction increases. In some others, qualitative AHP outperforms standard AHP and vice-versa. Hence, the results derived from the computational experiments lead to some interesting observations, when the number of alternatives is less than 10 .

In all these results, problems with less alternatives yielded fewer ranking contradictions than problems with more alternatives. As it can be seen, the number of criteria did not play a prime role. Hence, the results derived from the computational experiments lead to some interesting observations. First, none of the belief MCDM methods examined in this research is perfectly effective in terms of all the combinations. The results indicate that each method yields different percentages of contradiction. Especially, qualitative method yield to better results in most cases when both the number of alternatives and criteria is less than 10 .

\subsection{Catering selection problem}

The presented methods are illustrated with an example problem taken from literature: Catering selection problem. This latter is a MCDM problem which includes both qualitative and quantitative factors. In order to select the best catering firms it is necessary to make a tradeoff between these tangible and intangible factors some of which may be conflict. The objective of catering selection is to identify firms with the highest potential for meeting customers' needs consistently and at an acceptable cost.

In this section, we apply the same problem proposed by [18]. Thus, we consider a "problem of catering firms in Turkey": a case study of the application of the fuzzy AHP to the selection of best catering firms.

The companies of catering service sector in Turkey have to be very competitor. Their customers change frequently their supplier of catering contractor, because it is easy to replace them when a complaint or nonconformity happens, and there are too many companies in the sector. Three catering firms, Durusu $(D)$, Mertol $(M)$ and, Afiyetle $(A)$ were compared to select the best catering firm among the three.

The candidate criteria determined by the questionnaire were hygiene $(H)$, quality of meal (ingredients) $(Q M)$, and quality of service $(Q S)$. The Figure 6 summarizes our decision making problem.

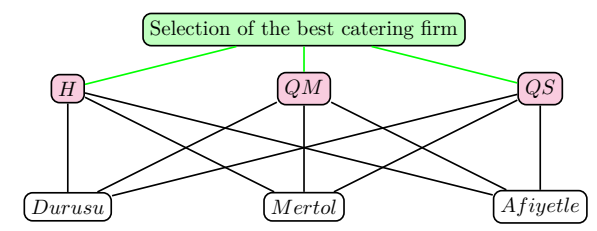

Figure 6: Hierarchy of catering firm selection problem

The hierarchical structure is simplified by the uncertain and qualitative methods and the sub-criteria in total are removed from decision analysis. In this situation, it is no wonder that the extent analysis method makes a wrong decision and he is concentrated on the three main criteria. The removal of the sub-criteria from decision analysis will not affect the final result. This problem has been solved using the presented AHP methods, and we have obtained the results shown in Figure 7. The three models recommended "Durusu" as the best alternative since it has the highest values.

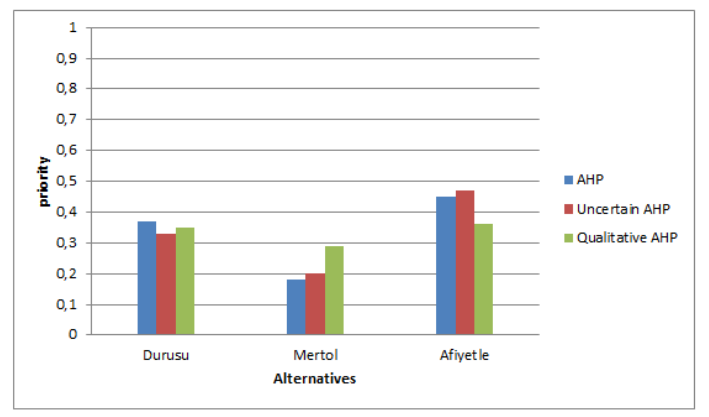

Figure 7: Catering selection problem 
For the sake of comparison, we compare our results to those obtained using Fuzzy AHP [18]. We have priority of $D=0.21$, priority of $A=0.69$ and priority of $M=0.10$. Using our approaches, we have obtained the alternatives $M$ and $A$ have nearly the same importance, especially using qualitative AHP. In addition, using the three models, the alternative $M$ is the worst one. Furthermore, the obtained results have been also affirmed by Wang et al. [19]. They have proved that alternative $M$ is the worst one.

\section{Conclusion}

Multi criteria Decision Making methods are gaining importance as potential tools for analyzing complex real problems due to their inherent ability to judge different alternatives on various criteria for possible selection of the best alternative. These alternatives may be further explored in-depth for their final implementation.

The objective of this present work was to evaluate the performance of three extended AHP methods using a series of randomly generated data. Results include what factors that affect the difference between these approaches most and in what way they affect the difference.

As a future work, we suggest to apply these introduced approaches on real application problems and to study their performance and applicability.

\section{References}

[1] M. Zeleny. Multiple Criteria Decision Making. McGraw-Hill Book Company, 1982.

[2] B. Roy. The outranking approach and the foundations of ELECTRE methods. Theory and Decision, 31:49-73, 1991.

[3] T. Saaty. A scaling method for priorities in hierarchical structures. Journal of Mathematical Psychology, 15:234-281, 1977.

[4] T. Saaty. The Analytic Hierarchy Process. McGraw-Hill, New-York, 1980.

[5] P.J.M. Van Laarhoven and W. Pedrycz. A fuzzy extension of Saaty's priority theory. Fuzzy Sets and Systems, 11:199-227, 1983.

[6] L. V. Utkin. A new ranking procedure by incomplete pairwise comparisons using preference subsets. Intelligent Data Analysis, 13(2):229-241, 2009.

[7] M. Beynon, B. Curry, and P. Morgan. The Dempster-Shafer theory of evidence: An alternative approach to multicriteria decision modelling. OMEGA, 28(1):37-50, 2000.

[8] A. Ennaceur, Z. Elouedi, and E. Lefevre. An extension of the analytic hierarchy process method under the belief function framework. Proceedings of the International Conference on Information Processing and Manage- ment of Uncertainty in Knowledge-Based Systems (IPMU), pages 233-242, 2014.

[9] A. Ennaceur, Z. Elouedi, and E. Lefevre. Multi-criteria decision making method with belief preference relations. International Journal of Uncertainty, Fuzziness and KnowledgeBased Systems, 22(4):573-590, 2014.

[10] Ph. Smets and R. Kennes. The Transferable Belief Model. Artificial Intelligence, 66:191234, 1994.

[11] G. Shafer. A Mathematical Theory of Evidence. Princeton University Press, 1976.

[12] N. Pal, J. Bezdek, and R. Hemasinha. Uncertainty measures for evidential reasoning I: A review. International Journal of Approximate Reasoning, 7:165-183, 1992.

[13] P. Smets. The application of the Transferable Belief Model to diagnostic problems. International Journal of Intelligent Systems, 13:127158, 1998.

[14] A. Ennaceur, Z. Elouedi, and E. Lefevre. Modeling expert preference using the qualitative belief function framework. Proceedings of the International Conference on Intelligent Systems Design and Applications (ISDA), pages 98-103, 2013.

[15] A. Ennaceur, Z. Elouedi, and E. Lefevre. Modeling qualitative assessments under the belief function framework. Proceedings of the International Conference on Belief Functions, pages 171-180, 2014.

[16] E. Triantaphyllou and S. H. Mann. An examination of the effectiveness of multi-dimensional decision-making methods: A decision-making paradox. Decision Support Systems, 5(3):303$312,1989$.

[17] E. Triantaphyllou and C. T. Lin. Development and evaluation of five fuzzy multiattribute decision-making methods. International Journal of Approximate Reasoning, 14(4):281-310, 1996.

[18] C. Kahraman, U. Cebecia, and D. Ruan's. Multi-attribute comparison of catering service companies using fuzzy AHP: The case of Turkey. International Journal of Production Economics, 87:171-184, 2004.

[19] Y. M. Wang, T. M. Elhag, and Z.S. Z. S. Hua. A modified fuzzy logarithmic least squares method for fuzzy Analytic Hierarchy Process. Fuzzy Sets and Systems, 157:3055-3071, 2006. 